\title{
A critical view of the general public's awareness and physicians' opinion of the trends and potential pitfalls of genetic testing in Greece
}

Yuan Mai ${ }^{1, *}$, Theodora Koromila ${ }^{2, *}$, Aggeliki Sagia ${ }^{1, *}$, David N. Cooper ${ }^{3}$, Georgios

Vlachopoulos ${ }^{4}$, George Lagoumintzis ${ }^{5}$, Panagoula Kollia ${ }^{2}$, Konstantinos Poulas ${ }^{1}$, Vlassios Stathakopoulos ${ }^{6}$, George P. Patrinos ${ }^{1, \#}$

${ }^{1}$ University of Patras, School of Health Sciences, Department of Pharmacy, Patras, Greece; ${ }^{2}$ National and Kapodistrian University of Athens, School of Physical Sciences, Department of Biology, Athens, Greece; ${ }^{3}$ Institute of Medical Genetics, School of Medicine, Cardiff University, Cardiff, United Kingdom; ${ }^{4}$ University of Patras, School of Medicine, Department of Medical Physics, Patras, Greece; ${ }^{5}$ Department of Optics and Optometry, School of Sciences of Health and Care, Technological Educational Institute of Patras; ${ }^{6}$ Department of Marketing and Communication, Athens University of Economics and Business, Athens, Greece;

* These authors contributed equally to this work

Running head: General public's awareness and physicians' opinion of genetic testing in Greece

\# Corresponding author at:

Department of Pharmacy, School of Health Sciences, University of Patras,

University Campus, Rion, GR-26504, Patras, Greece

Telephone/Fax: +30-2610-969.834, E-mail: gpatrinos@ upatras.gr 


\section{Executive Summary}

- Introduction: In the post-genomic era, we are witnessing significant advances in the functional decipherment of the human genome sequence that have been made possible by new technological developments in the field of genomic medicine. This, in turn, has facilitated the incorporation of genetic testing services into mainstream clinical practice. However, in many European countries, there is very little knowledge as to how either the general public or medical practitioners perceive genetics and genetic testing services.

- Purpose of the study and experimental approach: For this reason, we have initiated a pilot nationwide survey involving 1,717 individuals from the general population in Greece, divided according to age and residence, in order to better understand how the general public perceives genetic testing. At the same time, we performed a similar survey of 496 Greek physicians.

- Results: Our analysis indicated that a significant number of respondents from the general public are aware, at least in principle, of the nature of DNA and genetic disorders, as well as the potential benefits of genetic testing. Moreover, a large proportion of the respondents were willing to undergo genetic testing even if the costs of analysis would not be reimbursed. Perhaps surprisingly, only a relatively small proportion of the general public has actually been advised to undergo genetic testing, either by relatives or physicians.

- Also, a large proportion of the physicians who participated in this survey believe that the regulatory and legal frameworks that govern the provision of genetic testing services in Greece are rather weak. The vast majority of both the general public and 
physicians strongly opposes direct-access genetic testing, and would generally prefer referral to be from a physician rather than from a pharmacist.

- Conclusions: Overall, these results provide the basis for an assessment of the views of the general public and physicians on genetics and genetic testing services in Greece. These surveys could be readily replicated in other populations.

\section{Key Words:}

Genetic testing, general public awareness, physicians' opinion, molecular genetics, pharmacogenomics, education, health care, insurance costs reimbursement 


\section{Abstract}

Aim: Progress in deciphering the functionality of the human genome sequence in the wake of technological advances in the field of genomic medicine have dramatically reduced the overall costs of genetic analysis, thereby facilitating the incorporation of genetic testing services into mainstream clinical practice. Although Greek genetic testing laboratories offer a variety of different genetic tests, relatively little is known about how either the general public or medical practitioners perceive genetic testing services.

Materials and Methods: We have therefore performed a nationwide survey of the views of a total of 1,717 individuals from the general public, divided in three age groups from all over Greece, and residing in large and smaller cities and villages, in order to acquire a better understanding as to how they perceive genetic testing. We also canvassed the opinions of 496 medical practitioners with regard to genetic testing services in a separate survey that addressed similar issues.

Results: Our subsequent analysis indicated that a large proportion of the general public is aware of the nature of DNA, genetic disorders and the potential benefits of genetic testing, although this proportion declines steadily with age. Further, a large proportion of the interviewed sample would be willing to undergo genetic testing even if the costs of analysis were not covered by healthcare insurance. However, a relatively small proportion of the general public has actually been advised to undergo genetic testing, either by relatives or physicians. Most physicians believe that the regulatory and legal framework that governs genetic testing services in Greece is rather weak. Interestingly, the vast majority of the general public strongly opposes direct-access genetic testing, and most would prefer referral to be from a physician rather than from a pharmacist. 
Conclusions: Overall, our results provide a critical evaluation of the views of the general public with regard to genetics and genetic testing services in Greece and should serve as a model for replication in other populations. 


\section{Introduction}

Personalized or genomic medicine refers to the exploitation of genomic information in the context of guiding medical decision-making. Examination of an individual's genome sequence can, in principle at least, enable physicians to make assessments of disease risk and arrive at decisions regarding treatment regimens. At the same time, a number of health and disease states can now be identified by distinct genotypes and/or gene expression patterns. Hence, these molecular fingerprints can be exploited to stratify patient populations and to elucidate the pathogenesis of genetic disorders on a genome-wide basis $[1,2]$. We are entering an age in which individualized health care has become a reality by dint of taking each person's unique genomic profile into consideration alongside their clinical profile [3]. Our new-found knowledge of the molecular basis of many monogenic and complex disorders can be exploited not only in order to optimize preventive medicine strategies but also to personalize conventional therapeutic interventions, either at an early stage in the onset of the genetic disorder or pre-symptomatically, leading to unprecedented opportunities for the customization of patient care [4].

Unfortunately, among the general public, awareness may often be lacking with respect to genetics and its impact on society. Similarly, physicians, who are responsible for delivering these services to the general public, can have a relatively poor perception of certain issues pertaining to genomic medicine and its potential to fine-tune conventional medical interventions to the individual patient's genomic profile. As a result, the landscape of genetic testing services is still poorly developed in many parts of Europe and the United States despite courageous efforts to harmonize genetic testing services (e.g. EuroGenTest [101]; OrphaNet [102]). Thus, at the dawn of the genomic medicine era, understanding the general public's perception, as well as physicians' opinions, with respect to the potential societal and individual benefits (but also the problems and pitfalls) of genetic testing, has 
become an urgent goal. For this reason, we have initiated nationwide surveys to ascertain patients' and physicians' views of the genetic testing services currently available in Greece, aiming to (i) understand both the wishes and needs of patients and physicians with regard to the genetic testing industry and (ii) identify regulatory deficiencies and gaps in the existing legal provision that could be rectified by appropriate legislation.

We have previously reported our results from a nationwide survey of various private genetic testing providers in Greece [5]. Here, we report our findings from a survey of the general public's perception and physicians' opinion of various issues pertaining to genetics and its impact on society at large. Our study provides a model that can be replicated in other European countries with the ultimate aim of improving the public understanding of genetics and genetic testing, and facilitating the incorporation of genomic medicine into everyday clinical practice.

\section{Methods}

\section{Research design}

A cross-sectional survey design was used for this research study which was conducted between June 2009 and September 2010. We formulated two independent questionnaires (see Supplementary information) from which the data on individual perceptions of genetic testing and pharmacogenomics were generated.

In both questionnaires, non-random sampling was employed. The first questionnaire was personally given to 1,717 participants from the general public, that were selected from four major cities in Greece (Athens, Patras, Thessaloniki, Larissa), two small cities (fewer than 50,000 inhabitants) and two villages, including individuals with different occupations and ages. This questionnaire contained two main sections: the first part requested information such as age, gender and place of residence, whereas the second part 
contained 9 questions regarding various aspects of genetics, such as awareness of and personal opinion about genetics, genetic tests and the use of pharmacogenomic testing from health care providers. The second questionnaire was distributed to 496 physicians from all medical specialties that attended the national (Greek) medical conference in May 2010. This approach was taken to ensure a truly broad coverage of physicians from all specialties and from all geographical regions within the country. Again, this questionnaire contained two sections, the first pertaining to age and gender whilst the second posed five questions in order to solicit the respondents' opinions on the various potential benefits and pitfalls of genetic testing (see Supplementary information). We provided the necessary clarifications to questions posed by the survey respondents when required to do so, particularly in the case of the general public, in order to ensure that a valid response was given to each question.

\section{Measures}

The surveys provided the prospective data for this study. The dependent variables were derived from the questions in both surveys, scored using a binary model $(0=$ No, $1=$ Yes), whilst the independent variables comprised the demographic characteristics of respondents, particularly their age, gender and their place of residence.

\section{Statistical analysis}

All statistical analyses were performed using the Statistical Package for the Social Sciences, version 17.0 (SPSS Inc., Chicago, IL, USA). Frequency tables were obtained and statistical analysis was performed using the chi-square test. We also assessed the data for completeness and frequency distributions. Mean values, standard deviations, and percentages were computed to describe the distribution of independent variables. Cross- 
tabulation tables (contingency tables) were created to display the relationship between two or more (nominal or ordinal) variables using the chi-square test. Probabilities of less than 0.05 were considered statistically significant, when testing null hypotheses.

\section{Results}

The overall sample sizes and characteristics of the surveyed groups are shown in Table 1. Every effort was made in the context of both the general public and physicians' groups to be representative of the general public and medical practitioners' populations, respectively, in terms of both their gender and age. In the case of the general public, only adult respondents were surveyed. The distribution of the general public regarding their place of residence broadly followed Greek demographics [103]. The questionnaires aimed to ascertain the opinions of the general public and physicians on the following three issues: (a) awareness of genetics and genetic testing, (b) access, and various other issues pertaining to, genetic testing, and (c) direct-access genetic testing.

\section{Awareness of genetics and genetic testing}

We first attempted to critically evaluate the degree of education and overall awareness of the general public with respect to issues pertaining to genetics and genetic testing for both common and multifactorial genetic disorders. A significant proportion of the general public was found to be aware of the existence of DNA, the genetic material (GP-Q1), its biological role (GP-Q2), the main sources of DNA (GP-Q3) and the existence of public and private genetic testing laboratories (GP-Q4; Table 2); these elements were measured subjectively, as stated by the individuals who responded to the survey. As expected, there were significant differences when the respondents were subdivided according to their place of residence and their age. Indeed, the number of positive 
responses to these questions was inversely proportional to the age of the respondents, highlighting the fact that the general population is fairly uninformed about genetics. The same trend was also noted when the respondents were classified according to their place of residence, indicating reduced access of inhabitants of smaller cities and villages to information on genetics and genetic testing. Altogether, $84.3 \%$ of the general public expressed their willingness in principle to undergo genetic testing (Table 2).

\section{Access and various other issues pertaining to genetic testing}

One important parameter in personalized medicine is access to genetic testing for both common and multifactorial genetic disorders. Our surveys of both the general public and physicians were designed to address this aspect. From the physicians' questionnaire, we found that $74.5 \%$ would themselves be willing, at least in principle, to undergo genetic testing (Fig. 1A). However, a significantly lower percentage $(48.5 \%)$ had encouraged their patients to undergo genetic testing (Fig. 1B). More specifically, $42.1 \%$ had encouraged their patients to undertake a genetic test for a monogenic or multifactorial disorder, $30 \%$ a cytogenetic test and $16.1 \%$ a pharmacogenomic test (Fig. 1D). In the context of our own approach, molecular genetic testing was taken to refer both to monogenic and complex disease, the latter resulting from the interaction of genetic predisposition, negative lifestyle or other environmental factors. Despite the fact that both monogenic and complex disease involve the same sort of genetic analysis in the laboratory, the context and the interpretation of testing is very different in the two cases. These results are consistent with the nature of the genetic tests provided by private genetic testing laboratories in Greece, as indicated by our previous study [5]. Surprisingly, the general public indicated that only $9.5 \%$ had been encouraged to undertake a genetic test by their physicians, friends, relatives or a genetic laboratory representative (Fig. 1C). However, this proportion varied 
significantly when those who responded to the query were classified according to their place of residence, age or gender (Fig. 1E).

We then sought the general public's view as to their willingness to undertake a genetic test even if the costs would not be reimbursed by their insurance companies. It emerged that $54.8 \%$ of the general public would be willing to undertake a genetic test even if the costs would not be reimbursed (Fig. 2B). Again the proportion of those willing to take a genetic test for a monogenic or multifactorial disorder was significantly larger (94.1\%) than those willing to take a pharmacogenomic test, in a situation where the analysis costs would not be reimbursed (Fig. 2C). In concert with this finding, $77.3 \%$ of the physicians who responded to our questionnaire were of the opinion that the costs of genetic testing services should be reimbursed by insurance companies (Fig. 2A). However, it is noteworthy that a mere $11.9 \%$ of physicians believe that there is currently a satisfactory legal framework in Greece to cover aspects of genetic testing (Fig. 3) such as data privacy, written informed consent, genetic testing laboratory accreditation, regulation of genetic testing costs to avoid overpricing, and so on. This finding is again consistent with our previous finding indicating considerable variation in the accreditation of the various private genetic laboratories in Greece, the type of accreditation certificate, genetic testing pricing and the absence, in most cases, of a proper procedure to communicate results to the patients [5].

\section{Direct-access genetic testing}

Another very important aspect of genetic testing, with serious attendant ethical and (often) legal implications is direct-access testing, also known as direct-to-consumer genetic testing [6]. From our previous analysis, it is clear that at least one genetic service laboratory in Greece offers direct-access genetic testing as part of its public outreach 
strategy. In addition, buccal swab sampling kits for genetic testing are sold over the counter in at least one chain of pharmacies in Greece, while other pharmacies provide the same sampling kit upon request. We therefore sought the opinions of both the general public and physicians about direct-access genetic testing. Interestingly, only a very small proportion of the physicians $(12.7 \%$; Fig. 4A) were in favour of direct-access genetic testing, although this proportion was slightly higher among the general public $(17.9 \%$, $p=0.001$; Fig. 4C). Of those individuals from the general public who were against directaccess genetic testing $(82.1 \%)$, the vast majority wanted a physician to direct them to genetic testing services and then to explain the test results (96.6\%; Fig. 4D), whereas only $20.4 \%$ wanted a pharmacist to refer them to a genetic testing laboratory. These proportions varied significantly when the responders were classified according to their place of residence (Fig. 5), underlining the fact that only $7.7 \%$ of the responders who were living in a village favoured direct-access genetic testing services $(\mathrm{p}=0.036)$. Similarly, according to the physicians who were against direct-access genetic testing, the vast majority (89.7\%) believed that a physician should refer patients and/or interested individuals to a genetic testing laboratory whilst only 5\% believed that a pharmacist should be allowed to perform this task (Fig. 4B).

\section{Discussion}

The steady increase in the availability of genetic tests is a direct result of the exponential rate of discoveries in the field of human genomics, the technology now available for genome analysis [7], and our burgeoning knowledge of genotype-phenotype correlations. According to one estimate, more than 700.000 genetic tests are performed in Europe annually in both public and private genetic laboratories [8], and this is expected to increase in the years to come. In contrast to the United States, in Europe there are 
significant differences between individual countries, even between European Union member states, regarding genetic testing services. In other words, in some countries, there are established regulatory frameworks and provisions for genetic testing services, whereas in others the area is still not properly regulated (for an overview of the existing regulatory frameworks on genetic testing services, see the European Society of Human Genetics website [104]). Although there has been some attempt to harmonize genetic testing practices across Europe, an in-depth analysis, based upon comprehensive surveys of the current situation in European countries is still lacking. Presently, only a handful of studies have been performed in European populations to assess the attitude of the general public towards genetics and genetic testing services, namely in Finland [9], Germany [10] and Russia [11].

The present study complements our previous work on the private genetic testing environment in Greece [5], by attempting to explore how both the general public and medical practitioners perceive genetics and genetic testing services in the country. It was designed to explore how the Greek general public and their attending physicians perceive genetics and genetic testing, what their opinions are with respect to the regulatory and legal frameworks that oversee these services and what they think about the concept of directaccess genetic testing which has recently gained significant popularity. These surveys are not only among the very first of their kind performed in Europe, namely Finland and Germany, but also paid special attention (for the first time in Europe) to attitudes to pharmacogenomic testing since this emerging discipline is anticipated to have a central role in translational medicine in the future.

Our surveys included a large number of participants from the general public and physicians. We opted to carry out personal interviews rather than acquire information through electronic surveys since, from our own experience, the latter approach would not 
have yielded a satisfactory number of responses, particularly from older people and those living in smaller cities and villages who are likely to be less computer literate. Moreover, it would have probably introduced bias since those people who are most computer literate are also likely to be the most informed about genetic testing for a variety of reasons. Participants from the general public were therefore approached in several public places, such as pharmacies, supermarkets, cafés and restaurants, while physicians were selected while attending a major national medical conference in Athens. We fully appreciate that few of our questions required a simple "yes/no" answer, a study design which may not always provide the most useful insights when seeking to identify whether the public is aware of some specific fact or issue. On the other hand, we wished to avoid eliciting stereotypical responses and kept the questionnaire simple and easy to answer in order to encourage maximum participation on the part of our respondents, particularly the main target groups.

\section{Overall perception of genetics and genetic testing}

Our first attempt was to critically evaluate the degree of education and overall awareness of the general public with respect to issues pertaining to genetics and genetic testing. As indicated in Table 2, the majority of the general public was aware of the nature and role of the genetic material, as well as the various different sources from which an individual's DNA can be obtained. They were also well aware of the existence of both public and private genetic testing laboratories while $84.3 \%$ of the general public expressed their willingness to undergo genetic testing (Table 2). This proportion was markedly similar to that observed for a similar sized urban Russian population, where $85 \%$ of 2000 respondents answered positively to a question about their own willingness to undergo predictive genetic testing for preventable health conditions [11]. However, only a small 
proportion of people have actually been encouraged to undergo genetic testing by a physician, a relative or even a laboratory representative (Figs. 1C, E). Intriguingly, this proportion is somewhat lower than the professed willingness of the physicians to direct their patients to genetic testing services, if deemed necessary (Fig. 1B). This finding can be explained by the fact that although physicians are willing in principle to recommend genetic testing to their patients, in practice they often fail to do so. One possible explanation would be a lack of understanding and/or poor education with respect to the potential benefits of genetic testing of monogenic and complex disorders, classical or molecular cytogenetics, and pharmacogenomics. This concurs with a previous study which indicated that only 5\% of prescribers of azathioprine had requested DNA testing for variants in the TPMT gene to determine the ability of their patients to respond to the treatment [12]. Furthermore, significant differences were observed among the responses of the general public according to their places of residence, their age and, in certain cases, their gender (Table 2), which can again be explained by a lack of awareness of genetics and issues pertaining to genetic testing. These data are comparable to a similar sized study conducted in Russia, indicating that gender and age significantly influenced responses of the 2000 respondents that participated in this survey [11]. Our results also indicated that genetic tests for a monogenic or multifactorial disorder are strongly preferred over cytogenetic and pharmacogenomics tests (Fig. 1D), as indicated by the physicians' responses; these findings are concordant with our companion study of private genetic testing laboratories in Greece [5]. Similar results have also been reported from a comparable study in the UK, albeit involving a significantly smaller number of individuals [13].

The reimbursement of genetic testing costs by insurance companies is another important parameter of genetic testing to consider. The lack of any reimbursement could 
discourage interested parties from undergoing genetic testing, especially when costs are rather high or the patients are from a low-income bracket. A significant proportion $(54.8 \%)$ of the general public expressed their willingness to take a genetic test even if the costs would not be reimbursed (Fig. 2B), and again a preference for genetic testing for a monogenic or multifactorial disorder over pharmacogenomic testing was evident (Fig. 2C). Of course, this proportion is critically dependent on the health benefits, as these are perceived by the patient, and hence the latter percentage is likely to be smaller in relation to those tests that do not provide such information, e.g. pharmacogenomic tests predicting drug toxicity versus efficacy, respectively. These findings contrast with those from a recent survey in Canada which indicated that very few respondents were willing to pay for genetic testing to acquire information about genetic factors related to clinical disorders and $62 \%$ indicated that the public healthcare system should reimburse these tests [14]. It should be noted that the latter survey performed in Canada distinguished genetic tests with the type of information to be gained from it (such as genetic factors related to manageable conditions or serious, unpreventable disease, etc) and specified how much one is willing to pay (in cash brackets), and as such is not directly comparable to our present study.

Importantly, over $75 \%$ of the physicians questioned thought that the costs of genetic testing services should be reimbursed by insurance companies (Fig. 2A). Our questionnaire that was addressed to physicians did not distinguish between genetic testing for inherited disorders and pharmacogenomic testing; it would therefore be interesting to see if physicians differ from the general public in terms of their thinking on this issue. These findings should constitute a major driving force behind efforts to establish the necessary regulatory framework so that genetic testing costs can be reimbursed as of right. In their responses, the physicians emphasized the lack of a satisfactory legal framework to cover genetic testing (Fig. 3), such as the accreditation of genetic testing laboratories, data 
privacy, written informed consent and the regulation of genetic testing costs. In Greece, genetic testing services are mainly regulated through the legal framework that applies to the Greek national health care system as a whole and there are no dedicated laws specifically intended to cover genetic testing services. The regulations on patient rights are readily applicable as rights of genetic services users. The authority of the Greek Bioethics Committee is restricted to provide some important recommendations that complement the existing legislation. Our companion study [5] demonstrated that there is considerable variability in terms of (i) the accreditation of the various private genetic laboratories in Greece, (ii) the nature of their accreditation certificate, (iii) the cost of genetic testing and (iv) the proper means (or not, in most cases!) to communicate test results to the patients. There is a clear gap in current Greek legislation regarding direct-access genetic testing (see also below). Indeed, the Hellenic Society of Medical Geneticists [105] (content in Greek) and the Hellenic Bioscientists Association [106] (content in Greek) have both published warnings about direct-access testing services being offered by Greek private genetic laboratories (using call centers or advertising these tests over the Internet), stressing that these are highly specialized tests whose potential benefits and results cannot possibly be communicated by these means and by people who have not received the appropriate training.

\section{Direct-access genetic testing and society}

Direct-access genetic testing represents a very controversial issue with serious ethical and societal implications [15]. More than 1,000 genome variants are associated with susceptibility to genetic disorders and as a result, since 2007, an increasing number of genetic tests for common disorders and 'predictive markers' are available, most of which lack sufficient evidence of clinical validity, any proper meta-analysis of the marker(s) in 
question, and hence their utility in a clinical setting is doubtful [16]. These tests can be purchased via the Internet or over the counter in pharmacies in the United States and certain European countries, without the need for a medical specialist or biomedical scientist as intermediary. The results of these tests may therefore confuse the purchasers, and may falsely cause concern or even distress, or conversely provide false reassurance, while the lack of proper communication of the test results by a medical specialist deprives people of an adequate explanation for the potential consequences of the test result or of possible courses of remedial action in relation to their health. In other words, taking such tests may simply be a waste of money, thereby negatively impacting upon the public's opinion and diminishing their trust in genetic testing as used for bona fide medical purposes [17].

Our companion survey to private genetic laboratories indicated that at least one genetic laboratory in Greece offers direct-access genetic testing services [5], which had unfortunately not responded to our survey. Since direct-access genetic testing is a rather controversial issue and very few studies have previously been performed to canvass the general public's and physicians' opinions, we decided to include this topic in our surveys. Our results indicate that very few physicians (12.7\%) favour direct-access testing (Fig. 4A), although a slightly larger proportion of the general public hold the same view (17.9\%; Fig. 4C). In the latter case, this percentage was significantly higher compared to that obtained from a study performed in the UK [18], indicating that only 5\% of the respondents would be willing to undertake such a test, even if the price were less than 250 GBP. In the same study, a surprising 50\% responded positively to the hypothetical question as to whether they would be willing to undertake such a test even if it were offered free of charge. These data concur with similar results from the United States [19,20], indicating that individuals undergoing direct-access genetic testing have 
significant concerns about the entire process. Such information was not previously available in European populations [21], which was another important outcome from our surveys. Thus, the majority of both physicians and the general public are largely against direct-access genetic testing. This of course raises serious concerns with respect to the marketing channels that these laboratories employ to attract new customers. For example, advertising these tests through the Internet is the norm, whereas other marketing channels include cold-calls, ads in newspapers, or even information days at various venues (schools, municipal health centers, etc) from scientists collaborating with these laboratories. Preliminary data from our meta-analysis of several genes and DNA variants included in the tests offered indicate that, in the case of at least two genes and their accompanying variants, there is insufficient scientific evidence to include data pertaining to these genes/variants in the calculation of the overall risk (in preparation). This of course poses some serious concerns regarding the scientific accuracy of the results obtained.

Generally, those physicians and members of the general public who intimated that they were against direct-access genetic testing also indicated that they preferred a physician rather than a pharmacist to refer the interested parties to a genetic laboratory. Generally, physicians and pharmacists are the key interlocutors for the general public and this indeed was our reason for including them in the survey. In particular, $96.6 \%$ of the general public wished a physician to refer them to a genetic laboratory and to explain the test results to them, with a significantly smaller percentage (20.4\%) being content to go through a pharmacist. These percentages varied significantly when the responders were classified according to their place of residence (Fig. 5), emphasizing the fact that only $7.7 \%$ of the responders who were living in a village favoured direct-access genetic testing services. Similarly, with respect to the physicians who were against direct-access genetic testing, the vast majority $(89.7 \%)$ believed that only a physician should refer patients and 
interested individuals to a genetic testing laboratory whereas only $5 \%$ believed that it was appropriate for a pharmacist to undertake this task (Fig. 4B). These findings should be considered alongside the results of our companion study [5], which established that one Greek pharmacy group promotes genetic tests and sells DNA sampling kits to the public over the counter whereas other pharmacies are generally willing to order these sampling kits upon request. We have made enquiries with several pharmacies about the demand for these kits but it would appear that the demand is very low.

\section{Conclusions}

We provide here results from two nationwide surveys to assess the general public's awareness of, and physicians' opinion on, genetic testing services in Greece. Participants in our surveys were relatively well informed about genetics but, at the same time, more skeptical towards certain aspects of genetic testing, particularly direct-access genetic testing. In other words, it would appear that a better-informed general public harbours more critical views and is not necessarily more supportive of new genetics research and discoveries. Our results provide significant new insights into the potential benefits and pitfalls of genetic testing in Greece. Our future goal is to expand this study in order to acquire further insight into both public and physician's attitudes towards genetic testing so that legal issues and regulatory weaknesses may be addressed with the aim of ensuring that the field will come to be adequately and appropriately regulated. To this end, it is hoped that the existing gap between the overall provision of genetic testing in Europe and the US will somehow be bridged. Our study not only provides the basis for a critical appraisal of the genetic testing environment in Greece but also stands as a model for replication in other countries to assess the landscape of genetic testing services. 


\section{Acknowledgements}

We are indebted to the participants of our surveys, without whom this study would not have been possible. We also cordially thank Mrs. Kouskou Marianna, Koutloglou Sofia, Melissari Maria-Theodora, and Paliou Christina and Mr. Chris for their valuable assistance during this project. This work was partly funded by the Golden Helix Institute of Biomedical Research and by the University of Patras research budget.

\section{Financial disclosure}

The authors declare no competing financial interests.

\section{References}

1. Chen JM, Férec C, Cooper DN. Revealing the human mutome. Clin. Genet. 78(4), 310-320 (2010).

2. Cooper DN, Chen JM, Ball EV et al.: Genes, mutations, and human inherited disease at the dawn of the age of personalized genomics. Hum. Mutat. 31(6), 631-655 (2010).

3. Guttmacher AE, McGuire AL, Ponder B, Stefánsson K. Personalized genomic information: preparing for the future of genetic medicine. Nat. Rev. Genet. 11(2):161165 (2010).

** This article highlights various scientific, ethical and logistic issues that should be addressed so as to ensure that the potential benefits of personal genomic information outweigh the costs to both individuals and society.

4. Ginsburg GS, Willard HF. Genomic and personalized medicine: foundations and applications. Transl. Res. 154(6):277-287 (2009). 
5. Sagia A, Cooper DN, Poulas K, Stathakopoulos V, Patrinos GP. A critical appraisal of the private genetic and pharmacogenomic testing environment in Greece. Pers. Med. in press (2011).

* One of the first studies in a European country that attempts to appraise the current private genetic testing environment.

6. Hogarth S, Javitt G, Melzer D. The current landscape for direct-to-consumer genetic testing: legal, ethical, and policy issues. Annu. Rev. Genomics Hum. Genet. 9:161-182 (2008).

7. Metzker ML. Sequencing technologies - the next generation. Nat. Rev. Genet. 11(1):31-46 (2010).

8. Grimaldi KA, Look MP, Scioli GA, Clavero JC, Marinos S, Tagaris T. Personal genetics: regulatory framework in Europe from a service provider's perspective. Eur. J. Hum. Genet. 19(4):382-388 (2011).

9. Hietala M, Hakonen A, Aro AR, Niemelä P, Peltonen L, Aula P. Attitudes toward genetic testing among the general population and relatives of patients with a severe genetic disease: a survey from Finland. Am. J. Hum. Genet. 56(6):1493-1500 (1995).

10. Balck F, Berth H, Meyer W. Attitudes toward genetic testing in a German population. Genet. Test. Mol. Biomarkers 13(6):743-750 (2009).

11. Makeeva OA, Markova VV, Roses AD, Puzyrev VP. An epidemiologic-based survey of public attitudes towards predictive genetic testing in Russia. Pers. Med. 7(3), 291300 (2010).

12. Fargher EA, Tricker K, Newman W et al. Current use of pharmacogenetic testing: a national survey of thiopurine methyltransferase testing prior to azathioprine prescription. J. Clin. Pharm. Ther. 32(2):187-195 (2007). 
13. Fargher EA, Eddy C, Newman W et al. Patients' and healthcare professionals' views on pharmacogenetic testing and its future delivery in the NHS. Pharmacogenomics 8(11):1511-1519 (2007).

14. Ries NM, Hyde-Lay R, Caulfield T. Willingness to pay for genetic testing: a study of attitudes in a Canadian population. Public Health Genomics 13(5):292-300 (2010).

15. Caulfield T, Ries NM, Ray PN, Shuman C, Wilson B. Direct-to-consumer genetic testing: good, bad or benign? Clin. Genet. 77(2):101-105 (2010).

16. Khoury MJ, Coates RJ, Evans JP. Evidence-based classification of recommendations on use of genomic tests in clinical practice: dealing with insufficient evidence. Genet. Med. 12(11):680-683 (2010).

17. van El CG, Cornel MC. Genetic testing and common disorders in a public health framework. Eur. J. Hum. Genet. 19(4):377-381 (2011).

18. Cherkas LF, Harris JM, Levinson E, Spector TD, Prainsack B. A survey of UK public interest in internet-based personal genome testing. PLoS One 5(10), e13473 (2010).

19. Goddard KA, Duquette D, Zlot A et al. Public awareness and use of direct-toconsumer genetic tests: results from 3 state population-based surveys, 2006. Am. J. Public Health 99(3):442-445 (2009).

20. Bloss CS, Ornowski L, Silver E et al. Consumer perceptions of direct-to-consumer personalized genomic risk assessments. Genet. Med. 12(9):556-566 (2010).

* This study aimed to evaluate consumer perceptions of direct-access personalized genomic risk assessments and to assess the extent to which consumer characteristics may be associated with attitudes toward direct-access genetic testing.

21. Kricka LJ, Fortina P, Mai Y, Patrinos GP. Direct-access genetic testing: The view from Europe. Nat. Rev. Genet. in press, (2011). 
* This article compared the current landscape of direct-access genetic testing in the United States with the situation in Europe.

\section{Websites}

[101] EuroGenTest: http://www.eurogentest.org,

[102] OrphaNet: http://www.orpha.net

[103] Index Mundi: http://www.indexmundi.com/greece/demographics_profile.html

[104] European Society of Human Genetics: http://www.eshg.org

[105] Hellenic Association of Medical Geneticists: http://www.sige.gr

[106] Panhellenic Bioscientists Association: http://www.pev.gr 


\section{Figure legends}

\section{Figure 1}

Appraisal of access of physicians and the general public to genetic testing services. Outcome of the survey of physicians' willingness to personally undertake a genetic test (PQ3; A) and to direct their patients to genetic testing services (P-Q4; B), with a particular focus on genetic (P-Q4a), cytogenetic (P-Q4b) and pharmacogenomic testing (P-Q4c; D).

Outcome of the general public's feedback with respect to whether their physicians have recommended them to take a genetic test (GP-Q5; C) and detailed depiction of their positive replies in the various subgroups, regarding their place of residence, age and gender (E). *: $\mathrm{p}<0.5,{ }^{*}: \mathrm{p}<0.01$. Positive answers are depicted in green, negative answers in red.

\section{Figure 2}

Opinions of the physicians and the general public with regard to genetic testing and reimbursement of the corresponding costs. Physicians' opinion of the reimbursement of genetic testing costs $(\mathrm{P}-\mathrm{Q} 1 ; \mathbf{A})$, willingness of the general public to undergo genetic testing when analysis costs are not reimbursed by insurance companies (GP-Q8; B) and differences with respect to genetic and pharmacogenomic tests (GP-Q8a and GP-Q8b; C). Positive answers are depicted in green, negative answers in red.

\section{Figure 3}

Physicians' opinion with regard to the existence of the necessary legal framework covering genetic testing in Greece (P-Q2). Positive answers are depicted in green, negative answers in red. 


\section{Figure 4}

Opinions regarding direct-access genetic testing in Greece. Physicians’ overall views on the concept of direct-access genetic testing (P-Q5, P-Q5a and P-Q5b; A, B) and the corresponding view of the general public (GP-Q9, GP-Q9a and GP-Q9b; C, D). Positive answers are depicted in green, negative answers in red.

\section{Figure 5}

Differences in the views expressed by the general public with respect to direct-access genetic testing, according to their place of residence (see also text for details). 


\section{Table 1}

Survey sample composition and demographic elements. ${ }^{\text {a. }}$ Our questionnaires included adult respondents with a minimum age of 18 -years. ${ }^{\text {b. }}$ Fewer than 50,000 inhabitants.

\begin{tabular}{|c|c|c|c|}
\hline General Public (n) & 1,717 & Physicians (n) & 496 \\
\hline & $\%$ & & $\%$ \\
\hline \multicolumn{2}{|c|}{ Age (years) } & \multicolumn{2}{|c|}{ Age (years) } \\
\hline$<35^{a}$ & 32.6 & $<35^{a}$ & 32.6 \\
\hline $35-60$ & 49.5 & $35-60$ & 54.4 \\
\hline$>60$ & 17.9 & $>60$ & 11.9 \\
\hline \multicolumn{2}{|c|}{ Gender } & \multicolumn{2}{|c|}{ Gender } \\
\hline Male & 46.9 & Male & 48.3 \\
\hline Female & 53.1 & Female & 51.7 \\
\hline \multicolumn{4}{|c|}{ Place of residence } \\
\hline City & 63 & & \\
\hline Smaller City ${ }^{b}$ & 33.2 & & \\
\hline Village & 2.8 & & \\
\hline
\end{tabular}




\section{Table 2}

Critical evaluation of the awareness of the general public on DNA and genetic testing services. Statistically significant values are shown in boldface.

\begin{tabular}{|c|c|c|c|c|c|c|c|c|c|c|c|c|c|}
\hline & & GP-Q1 & p-value & GP-Q2 & p-value & GP-Q3 & $p$-value & GP-Q4 & p-value & GP-Q6 & p-value & GP-Q7 & p-value \\
\hline \multirow{3}{*}{$\begin{array}{l}\text { Place of } \\
\text { residence }\end{array}$} & City & 93.7 & 0.000 & 80.8 & 0.000 & 87.6 & 0.000 & 72.3 & 0.000 & 85 & 0.535 & 77.4 & 0.375 \\
\hline & Small city & 90 & 0.000 & 77.5 & 0.000 & 82.2 & 0.000 & 65.8 & 0.000 & 82.9 & 0.535 & 74.7 & 0.375 \\
\hline & Village & 61.5 & 0.000 & 16.9 & 0.000 & $\mathbf{0}$ & 0.000 & $\mathbf{0}$ & 0.000 & 83.1 & 0.535 & 80 & 0.375 \\
\hline \multirow{3}{*}{$\begin{array}{c}\text { Age } \\
\text { (years) }\end{array}$} & $<35$ & 94.6 & 0.000 & 85.5 & 0.000 & 92.1 & 0.000 & 81.7 & 0.000 & 87.1 & 0.066 & 74.4 & 0.133 \\
\hline & $35-60$ & 90.7 & 0.000 & 82.1 & 0.000 & 78.2 & 0.000 & 65 & 0.000 & 83.3 & 0.066 & 78.7 & 0.133 \\
\hline & $>60$ & 86.6 & 0.000 & 60 & 0.000 & 66.2 & 0.000 & 48.1 & 0.000 & 81.8 & 0.066 & 75 & 0.133 \\
\hline \multirow{2}{*}{ Sex } & Male & 88.9 & 0.001 & 74.8 & 0.017 & 80.3 & 0.027 & 63.9 & 0.004 & 81.6 & 0.004 & 74.6 & 0.068 \\
\hline & Female & 93.3 & 0.001 & 79.6 & 0.017 & 84.4 & 0.027 & 70.5 & 0.004 & 86.6 & 0.004 & 78.4 & 0.068 \\
\hline Overall & Positive & 91.3 & & 77.3 & & 82.5 & & 67.5 & & 84.3 & & 76.6 & \\
\hline
\end{tabular}

Abbreviations: GP-Q1-4,6,7: General public-Question1-4,6,7 